\title{
Histomorphology of pancreatic cancer in patients with inherited ATM serine/threonine kinase pathogenic variants
}

\author{
Danielle Hutchings ${ }^{1} \cdot$ Zhengdong Jiang $^{1,2} \cdot$ Michael Skaro ${ }^{1} \cdot$ Matthew J. Weiss ${ }^{3,4}$. Christopher L. Wolfgang ${ }^{3,4}$.

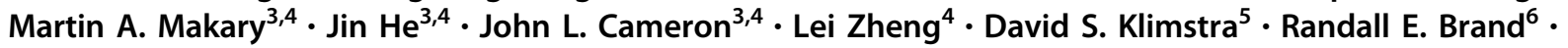 \\ Aatur D. Singhi ${ }^{7} \cdot$ Michael Goggins ${ }^{1} \cdot$ Alison P. Klein ${ }^{4} \cdot$ Nicholas J. Roberts $\mathbb{D}^{1,4} \cdot$ Ralph H. Hruban $^{1,4}$
}

Received: 25 January 2019 / Revised: 5 June 2019 / Accepted: 5 June 2019 / Published online: 8 July 2019

(C) The Author(s), under exclusive licence to United States \& Canadian Academy of Pathology 2019

\begin{abstract}
Germline pathogenic variants in the ATM serine/threonine kinase (ATM) gene are associated with an increased risk of pancreatic ductal adenocarcinoma. It is important to identify germline $A T M$ pathogenic variants in pancreatic cancer patients because these alterations are potentially targetable with chemotherapeutic drugs and/or radiation and have implications for other family members. As germline pathogenic variants in other genes have been associated with distinct histologic subtypes of pancreatic cancer, we studied the histomorphology of pancreatic cancer in 23 patients with germline ATM pathogenic variants. The histologic subtype was ductal adenocarcinoma in 19/23 (83\%) of the patients, adenosquamous carcinoma in 1/23 (4\%), and colloid (mucinous non-cystic) carcinoma in 3/23 (13\%). The percentage of colloid (mucinous non-cystic) carcinomas is higher than we have previously observed in patients with familial and sporadic pancreatic cancer (1 and $2 \%$ in prior reports, $p<0.01$ and $p<0.01$, respectively). Three carcinomas ( 2 colloid carcinomas, 1 ductal adenocarcinoma) arose in association with intraductal papillary mucinous neoplasms. Among the resected pancreata, non-invasive precursor lesions, including pancreatic intraepithelial neoplasia and incipient intraductal papillary mucinous neoplasms, were identified in $83 \%$. We conclude that pancreatic cancers in patients with germline ATM pathogenic variants are more frequently of colloid (mucinous non-cystic) morphology but are overall morphologically diverse supporting the utility of universal germline genetic testing for patients with pancreatic cancer.
\end{abstract}

\section{Introduction}

Pancreatic cancer is a particularly lethal form of cancer with a 5-year survival rate of only $9 \%$ [1]. In 2019, an estimated

Supplementary information The online version of this article (https:// doi.org/10.1038/s41379-019-0317-6) contains supplementary material, which is available to authorized users.

Nicholas J. Roberts nrobert8@jhmi.edu

$\triangle$ Ralph H. Hruban rhruban@jhmi.edu

1 Department of Pathology, the Sol Goldman Pancreatic Cancer Research Center, the Johns Hopkins University School of Medicine, Baltimore, MD, USA

2 Department of Hepatobiliary Surgery, First Affiliated Hospital of Xi'an Jiaotong University, Xi'an, Shaanxi, China

3 Department of Surgery, the Sol Goldman Pancreatic Cancer
56,770 people will be newly diagnosed with pancreatic cancer and 45,750 people will die of the disease in the United States [1]. One area of increasing importance in pancreatic cancer is the identification of germline alterations in cancer predisposition genes. To date, pathogenic variants in ATM, BRCA1, BRCA2, CDKN2A, CPA1, CPB1, PALB2, PRSS1, STK11, TP53, and the mismatch repair genes (MLH1, MLH2, PMS2, MSHO) are known to be associated

Research Center, the Johns Hopkins University School of Medicine, Baltimore, MD, USA

4 Department of Oncology, the Sol Goldman Pancreatic Cancer Research Center, the Johns Hopkins University School of Medicine, Baltimore, MD, USA

5 Department of Pathology, Memorial Sloan Kettering Cancer Center, New York, NY, USA

6 Department of Medicine, University of Pittsburgh Medical Center Health System, Pittsburgh, PA, USA

7 Department of Pathology, University of Pittsburgh Medical Center Health System, Pittsburgh, PA, USA 
with an increased risk of pancreatic ductal adenocarcinoma $[2,3]$. It is estimated that $4-10 \%$ of all pancreatic cancer patients will harbor a germline pathogenic variant in one of these genes [2, 4-8]. Identification of these variants are important for targeted treatment, screening and prevention of other cancers, and genetic counseling of families $[9,10]$.

The ATM serine/threonine kinase (ATM) gene was first recognized as the cause of ataxia telangiectasia, an inherited autosomal recessive disorder characterized by cerebellar ataxia, skin telangiectasia, immunodeficiency, and increased risk of cancer [11]. It was subsequently discovered that carriers of germline ATM pathogenic variants are at significantly increased risk of breast cancer [12]. Roberts et al. first described ATM as a pancreatic cancer susceptibility gene in familial pancreatic cancer patients in 2012 [13]. More recently, large-scale sequencing studies have found germline ATM pathogenic alterations in up to $3.3 \%$ of familial pancreatic cancer patients and $2.3 \%$ of pancreatic cancer patients unselected for family history [4, 5, 8, 14, 15]. Cancers with loss of ATM are important to identify for treatment purposes as they are potentially targetable with poly(ADP-ribose) polymerase 1 inhibitors, ATR serine/threonine kinase inhibitors, DNA-protein kinase catalytic subunit inhibitors, and radiation therapy $[9,10]$. The identification of a germline pathogenic ATM variant would also have obvious implications for the patient's other family members.

In some instances, tumor histomorphology may raise the suspicion that a cancer arose in the setting of a specific germline abnormality and may prompt further testing. For example, medullary carcinoma of the pancreas has been associated with pathogenic alterations in DNA mismatch repair genes [16-18]. The association of a tumor morphology with a specific deleterious germline abnormality is also important because it has the potential to play a role in the clarification of germline genetic variants of unknown significance for some patients. In this study, we describe the histomorphology and clinicopathologic features of pancreatic cancers from patients with germline $A T M$ pathogenic variants. In the absence of distinguishing pathology, our results support the 2019 updates to the National Comprehensive Cancer Network guidelines, which state that germline testing should be considered for all patients with pancreatic cancer [19].

\section{Materials and methods}

\section{Patient selection}

Study approval was obtained from the Johns Hopkins Institutional Review Board. Patients with pancreatic cancer resection and/or biopsy specimens, and germline ATM variants classified as pathogenic or likely pathogenic according to American College of Medical Genetics criteria were identified from patient medical records or previous research studies from The Johns Hopkins Hospital, Memorial Sloan Kettering Cancer Center, and University of Pittsburgh Medical Center and included in this study $[4,13,15,20]$.

For each patient, demographic data including age, sex, and race were obtained from pathology records. The electronic medical records were searched for clinical information including family history of cancer, personal history of other cancers, treatment with neoadjuvant and/or adjuvant chemotherapy and/or radiation, disease recurrence, and patient survival. Patients were categorized as alive with no evidence of disease, alive with disease if they had radiographic, pathologic or biochemical (elevated serum CA199) evidence of disease recurrence, deceased of disease, deceased of unrelated cause or deceased of unknown cause.

\section{Pathologic review}

For each patient, all available hematoxylin and eosin stained slides were reviewed from resection and/or biopsy specimens. Pancreatic carcinomas were classified by histologic subtype according to World Health Organization criteria [21]. The presence or absence of lymphovascular invasion, perineural invasion, and lymph node metastases was noted. Cases were reviewed for the presence or absence of neoplastic precursor lesions, such as pancreatic intraepithelial neoplasia and intraductal papillary mucinous neoplasm, which when present were classified and graded for dysplasia based on consensus recommendations [22]. Lesions were classified as incipient intraductal papillary mucinous neoplasms when they were $>0.5 \mathrm{~cm}$ but $<1.0 \mathrm{~cm}$ and had intestinal differentiation or long papillae [23]. Slides of background pancreas uninvolved by tumor were scanned on the Ventana iScan HT (Roche) at $\times 40$ magnification and analyzed in ImageScope v12.2.2.5015 (Aperio). The area of pancreas uninvolved by tumor was measured and the number of duct profiles containing neoplastic precursor lesions were counted. The density of precursor lesions per square centimeter $\left(\mathrm{cm}^{2}\right)$ of pancreatic parenchyma was calculated.

When applicable, treatment effect was scored according to the College of American Pathologists staging protocol for exocrine pancreas carcinomas $[24,25]$. In brief, no residual tumor was considered complete response, single cells or rare small groups of cells in a fibrotic background a marked response, residual cancer outgrown by fibrosis was moderate response, and extensive residual cancer was poor response. Gross descriptions were reviewed for tumor size and location. Tumors were staged according to the American Joint Committee on Cancer eighth edition [26]. 


\section{Statistical analysis}

To assess for differences in histologic subtypes of pancreatic cancer in unselected patients versus those with germline ATM pathogenic variants, a Fisher's exact test was used to compare our results to the histologic subtypes of patients with familial and sporadic pancreatic cancers reported by Singhi et al. [27]. Negative binomial regression was used to compare the number of precursor lesions per $\mathrm{cm}^{2}$ of pancreatic tissue in patients with germline $A T M$ pathogenic variants with previously reported familial and sporadic pancreatic cancer patients [28]. Comparisons of progression-free survival were conducted with a MantelCox log-rank test. Prism6 (GraphPad) was used for Fisher's exact and Mantel-Cox log-rank tests. SPSS (IBM) was used for negative binomial regression. In all tests, A $p$-value $<$ 0.05 was considered significant.

\section{Results}

\section{Patient characteristics}

Twenty-three patients with ATM germline variants classified as either pathogenic or likely pathogenic according to American College of Medical Genetics criteria were included in this study [20]. The patient characteristics and germline ATM pathogenic variants are presented in Table 1 . In summary, $52 \%$ were female, $89 \%$ were white, and $57 \%$ were aged 60 years or older. Nine (39\%) patients had germline nonsense variants in the ATM gene, seven (30\%) patients had germline frameshift variants, six (26\%) had germline splice site variants, and one (4\%) had a germline missense variant. Clinical history was available in 20 patients. Nine (45\%) of 20 patients had a reported previous personal history of other (non-pancreatic) cancer including 5 (25\%) with a history of breast cancer. Fifteen (75\%) of 20 patients had a known family history of cancer including $13(65 \%)$ with a family history of pancreatic and/or breast cancer. Five (25\%) patients lacked both a personal history of cancer and family history of pancreatic cancer.

\section{Gross and histomorphologic findings}

Twenty-two patients had resections available for review, whereas one had only a core biopsy available. The gross and histomorphologic findings are summarized in Table 2. Nineteen $(83 \%)$ of the cancers were ductal adenocarcinoma, three $(13 \%)$ were colloid (mucinous non-cystic) carcinoma, and one (4\%) was adenosquamous carcinoma (Fig. 1). Three carcinomas arose in association with intraductal papillary mucinous neoplasms, including two cases of colloid carcinoma with associated intestinal-type intraductal papillary mucinous neoplasm with high-grade dysplasia and one case of ductal adenocarcinoma with associated gastric foveolar-type intraductal papillary mucinous neoplasm with low-grade dysplasia. No definite precursor intraductal papillary mucinous neoplasm was identified in the third case of colloid carcinoma. Compared to sporadic and familial pancreatic cancer cases reported by Singhi et al. [27], carcinomas in patients with germline ATM pathogenic variants were more often colloid (mucinous non-cystic) carcinoma (familial $-p<0.01$; sporadic $-p<0.01$; Table 3 ). Among the 22 resections, the median tumor size was $2.8 \mathrm{~cm}$ (range $0.1-4.8 \mathrm{~cm}$ ) and $72 \%$ of the carcinomas were located in the head of the pancreas. One patient had two separate, discrete tumor nodules in the head of the pancreas measuring 2.0 and $0.9 \mathrm{~cm}$ (Patient 21, Table 1). Perineural and angiolymphatic invasion were observed in $91 \%$ and $59 \%$ of cases, respectively. Metastases to one or more lymph nodes were present in $68 \%$ of cases.

The pathologic stage and response to treatment (if applicable) for each patient is reported in Table 1. Of the seven patients treated with neoadjuvant therapy, six received neoadjuvant chemotherapy alone with five showing poor pathologic response to treatment (Patients $15,16,18,21$, and 22, Table 1) and one showing moderate response (Patient 17, Table 1). One patient was treated with a combination of radiation and chemotherapy and showed marked pathologic response to treatment (Patient 20, Table 1).

Histologic sections of pancreatic parenchyma not involved by invasive carcinoma were available for review in 18 out of 22 patients who underwent surgical resection. Non-invasive precursors including pancreatic intraepithelial neoplasia and incipient intraductal papillary mucinous neoplasms were identified in $15(83 \%)$ of 18 patients. Among the four (22\%) patients with incipient intraductal papillary mucinous neoplasms, all had ductal adenocarcinoma and gastric foveolar-type intraductal papillary mucinous neoplasms with low-grade dysplasia. Fifteen (83\%) patients had pancreatic intraepithelial neoplasia, all with low-grade dysplasia. For each case, the number of precursor lesions per square centimeters $\left(\mathrm{cm}^{2}\right)$ of pancreatic parenchyma were quantified. The median of uninvolved pancreas analyzed was 2.76 (range $0.27-10.3$ ) $\mathrm{cm}^{2}$ per case. The rate of total precursors, including low-grade pancreatic intraepithelial neoplasia lesions and low-grade incipient intraductal papillary mucinous neoplasms was 2 per $\mathrm{cm}^{2}$ (range 0-4 per $\mathrm{cm}^{2}$ ). There were no statistically significant differences between rate of total precursors in patients with germline $A T M$ pathogenic variants and previously reported familial and sporadic pancreatic cancer patients (Table 4) [28]. 


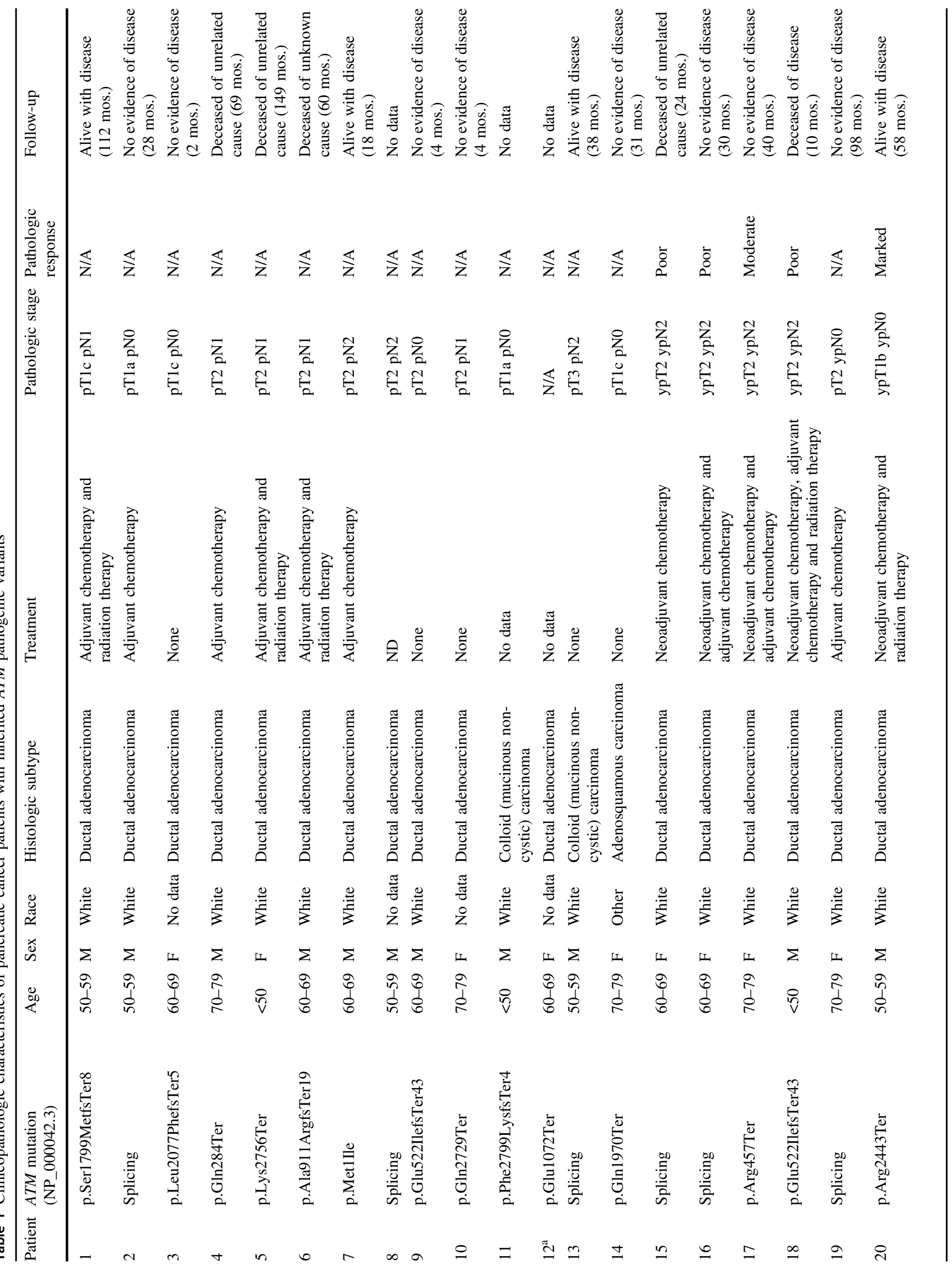




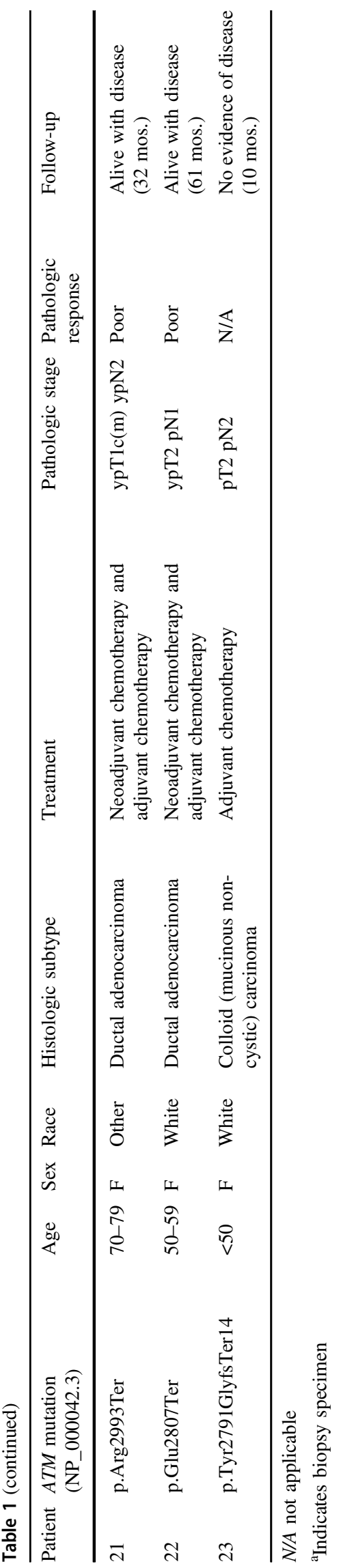

Table 2 Gross and histomorphologic characteristics of pancreatic cancers in patients with germline $A T M$ pathogenic variants

\begin{tabular}{lc}
\hline Tumor size, median $(n=22)$ & $2.8 \mathrm{~cm}($ range $0.1-4.8 \mathrm{~cm})$ \\
\hline Tumor site $(n=22)$ & $16(72)$ \\
Head, $n(\%)$ & $3(14)$ \\
Body, $n(\%)$ & $3(14)$ \\
Tail, $n(\%)$ & \\
Histologic subtype $(n=23)$ & $19(83)$ \\
$\quad$ Ductal adenocarcinoma, $n(\%)$ & $3(13)$ \\
Colloid (mucinous non-cystic) & $1(4)$ \\
carcinoma, $n(\%)$ & $2(9)$ \\
Adenosquamous carcinoma, $n(\%)$ & $20(91)$ \\
Perineural invasion $(n=22)$ & \\
Absent, $n(\%)$ & $9(41)$ \\
Present, $n(\%)$ & $13(59)$ \\
Angiolymphatic invasion $(n=22)$ & $7(32)$ \\
Absent, $n(\%)$ & $6(27)$ \\
Present, $n(\%)$ & $9(41)$ \\
Lymph node metastasis $(n=22)$ & \\
None, $n(\%)$ & \\
$1-3, n(\%)$ & \\
4 or more, $n(\%)$ & \\
\hline
\end{tabular}

\section{Clinical outcomes}

Clinical follow-up was available in 20 out of 23 patients and is presented in Table 1. All 20 patients with followup had surgical resection. One patient was enrolled in a pancreatic cyst screening program. Fifteen (75\%) were treated with neoadjuvant and/or adjuvant chemotherapy and/or radiation (Table 1). Overall, patients were followed for a median of 32 months (range 2-149 months). Five patients were followed until death and 15 patients were alive at the time of last follow-up (Table 1). The median progression-free survival for all patients was 51 months (range 2-149 months). The median progression-free survival time for those patients receiving neoadjuvant, adjuvant, neoadjuvant and adjuvant, and neoadjuvant and/or adjuvant was 40 months (range 23-57 months), 84 months (range 8-149 months), 51 months (range 4-51 months), and 51 months (range 4-149 months) respectively. The median progression-free survival for patients receiving surgery only was 35 months (range 2-35 months). There was no statistically significant difference between progression-free survival for patients receiving and not receiving neoadjuvant therapy and/or adjuvant therapy (Supplementary Figs. 1-4). 
Fig. 1 Morphologic spectrum of precursor lesions and pancreatic carcinomas in patients with germline ATM pathogenic variants. a Incipient intraductal papillary mucinous neoplasm, gastric foveolar-type with low-grade dysplasia.

b Well-differentiated invasive ductal adenocarcinoma. c Invasive ductal adenocarcinoma with clear cell features. d Invasive colloid (mucinous non-cystic) carcinoma. e Invasive adenosquamous carcinoma. f Invasive ductal adenocarcinoma with moderate treatment response.

Hematoxylin and eosin stain $\times 10(\mathbf{a}), \times 200(\mathbf{b}-\mathbf{d}, \mathbf{f})$, and $\times 400($ e)

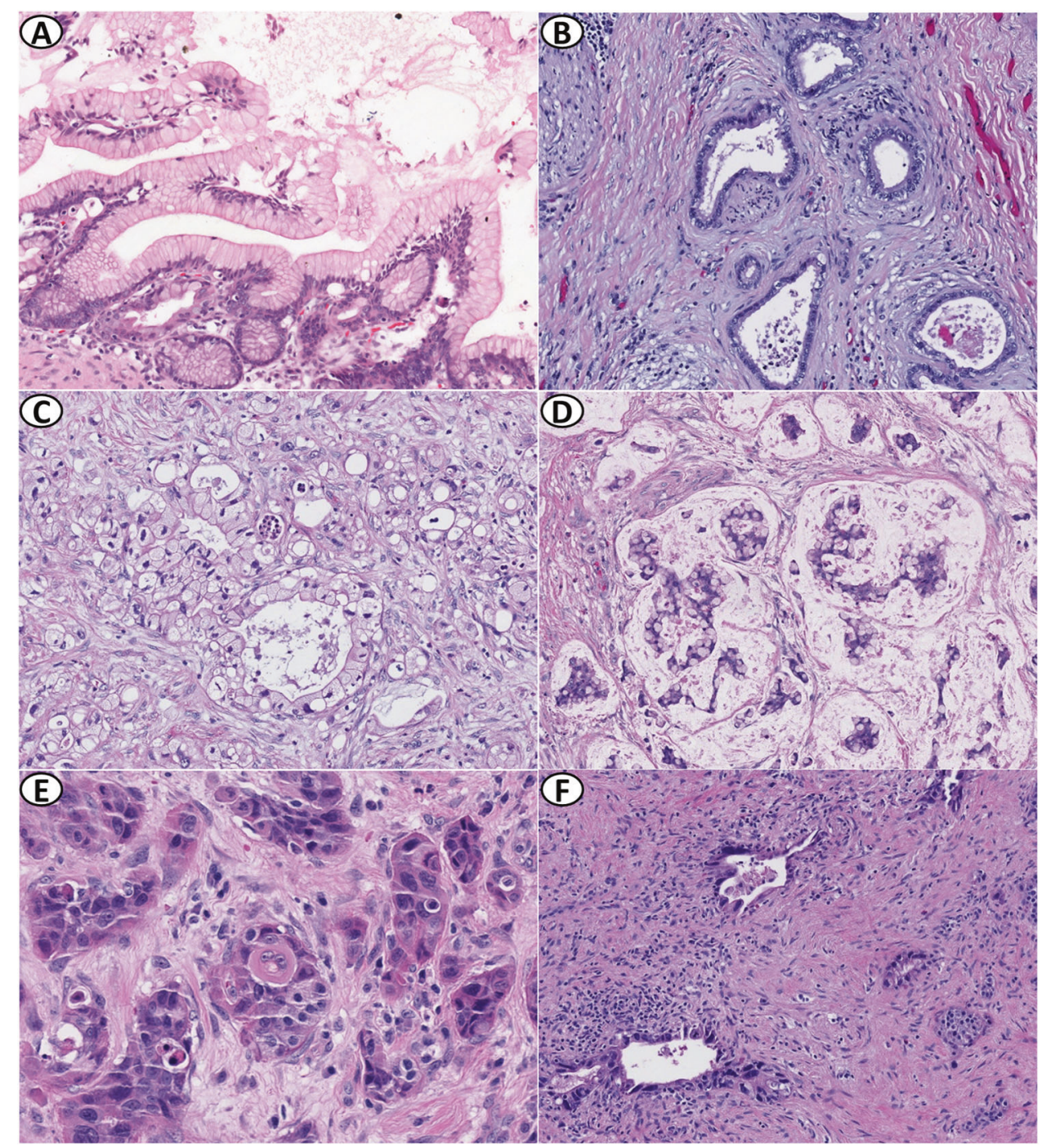

Table 3 Comparison of histologic subtype between patients with germline ATM pathogenic variants and familial or sporadic pancreatic cancer patients

\begin{tabular}{|c|c|c|c|c|c|}
\hline Histologic subtype & $\begin{array}{l}\text { Patients with germline } A T M \\
\text { pathogenic variants }(n=23)\end{array}$ & $\begin{array}{l}\text { Familial pancreatic cancer } \\
\text { patients }(n=519)\end{array}$ & $p$-value & $\begin{array}{l}\text { Sporadic pancreatic cancer } \\
\text { patients }(n=651)\end{array}$ & $p$-value \\
\hline Colloid carcinoma, $n(\%)$ & $3(13)$ & $4(1)$ & 0.002 & $10(2)$ & 0.008 \\
\hline $\begin{array}{l}\text { Other (non-colloid) } \\
\text { carcinoma, } n(\%)\end{array}$ & $20(87)$ & 515 (99) & & $621(98)$ & \\
\hline
\end{tabular}

Data for familial and sporadic pancreatic cancer patients from Singhi et al. [27]

\section{Discussion}

The identification of germline pathogenic variants in patients with pancreatic cancer is crucial to improving treatment and prevention of this deadly disease. Inherited ATM pathogenic variants are found in a small but significant subset of pancreatic cancer patients and are important since they are potentially targetable with selected drugs and may confer susceptibility to radiation therapy
$[9,10]$. In some situations, certain pathologic features may prompt germline testing. Perhaps the best example of this is the medullary phenotype which is associated with microsatellite instability and germline pathogenic variants in a DNA mismatch repair genes [16-18]. Moreover, the presence of distinctive histology could also provide clinical correlation with the results of germline testing and potentially aid in the interpretation of genetic variants of unknown significance. 
Table 4 Precursor lesions in patients with germline ATM pathogenic variants

\begin{tabular}{ll}
\hline & $\begin{array}{l}\text { Average number per } \\
\mathrm{cm}^{2}(n=18)^{\mathrm{a}}\end{array}$ \\
\hline $\begin{array}{l}\text { Total precursors } \\
\text { Low-grade incipient intraductal papillary } \\
\text { mucinous neoplasms }\end{array}$ & 2 \\
$\begin{array}{l}\text { Low-grade pancreatic intraepithelial } \\
\text { neoplasia }\end{array}$ & 2 \\
\hline
\end{tabular}

${ }^{a}$ Average number per $\mathrm{cm}^{2}$ rounded to nearest whole number

In this study, we examined the histomorphology of pancreatic cancers in patients with germline ATM pathogenic variants and found that the majority of patients (83\%) had conventional ductal adenocarcinoma, which lacked any distinctive histomorphologic characteristics. Interestingly, patients with a germline ATM pathogenic variant had more cancers with colloid (mucinous non-cystic) carcinoma histology than did previously reported sporadic or familial pancreatic cancer patients $(p<0.01$ and $p<0.01$ respectively). As the number of patients with colloid carcinoma is small, larger studies to validate these findings are warranted. However, colloid (mucinous non-cystic) carcinoma histology may help identify a subset of patients with germline ATM pathogenic variants.

A study by Shi et al. comparing non-invasive precursor lesions in patients with familial versus sporadic pancreatic carcinoma demonstrated that non-invasive precursor lesions are more common and more extensive in familial pancreatic cancer patients [28]. We did not observe a statistically significant difference between rate of total precursor lesions in background pancreatic parenchyma in patients with germline ATM pathogenic variants compared to previously reported sporadic and familial pancreatic cancer patients. However, power to detect such differences is limited by our study sample size. In addition, our patients were overall younger with a median age 60 years versus the mean age of 66 and 65 years in the familial and sporadic groups, respectively in Shi et al. [28].

Our patient cohort was also demographically and clinically diverse and included a subset of patients lacking characteristics commonly associated with inherited cancers such as family history [29]. A quarter of patients in our study lacked both a personal history of cancer and a family history of pancreatic cancer.

We found that the median progression-free survival for pancreatic cancer patients with germline ATM pathogenic variants was 51 months. By comparison, the mean survival of unselected patients with pancreatic cancer who underwent surgical resection at our institution was 19 months and 23 months in two prior studies [30, 31]. One patient, however, was enrolled in a pancreatic cyst screening program which can positively affect survival. Of note, patients in our cohort received variable neoadjuvant and/or adjuvant treatment. Interestingly, one patient received neoadjuvant combination chemotherapy/radiation and had a more significant pathologic treatment response than patients treated with chemotherapy alone. This appears consistent with evidence that ATM deficient pancreatic cancer cells are more susceptible to radiation [10]. However, there was no statistically significant difference in progression-free survival for patients receiving neoadjuvant and/or adjuvant treatment compared to those treated with surgery alone. Additional larger and controlled studies are needed to determine if germline ATM pathogenic variants portend a better prognosis.

We used American College of Medical Genetics criteria to classify variants and included patients with pathogenic or likely pathogenic variants in this study [20]. However, even variants classified as pathogenic or likely pathogenic may not always be deleterious to protein function. For example, variants in splice regions (which were found in three patients) are predicted to affect splicing using the human splice finder, however, functional testing of these variants to confirm the splicing defect affects the protein product would be helpful [32]. Demonstration of loss of ATM function or expression in vitro and segregation with disease in familial pancreatic cancer or ataxia telangiectasia families would further strengthen variant classification.

In summary, we present the clinicopathologic characteristics of pancreatic cancers in 23 patients with germline ATM pathogenic variants, the largest series to date. We found significantly more patients had colloid (mucinous non-cystic) carcinoma histology compared to previously published data from sporadic and familial pancreatic cancer patients. Otherwise, cancers in these patients were histomorphologically and clinically diverse, further supporting the 2019 National Comprehensive Cancer Network guidelines which recommend the consideration of germline genetic testing in all patients with pancreatic cancer [19].

Acknowledgements This work was supported by NIH/NCI P50 CA62924 and R00 CA190889, Susan Wojcicki and Dennis Troper, the Sol Goldman Pancreatic Cancer Research Center, the Art Creates Cures Foundation, the Rolfe Pancreatic Cancer Foundation, the Joseph C. Monastra Foundation, and the Gerald O Mann Charitable Foundation (Harriet and Allan Wulfstat, Trustees).

\section{Compliance with ethical standards}

Conflict of interest The authors declare that they have no conflict of interest.

Publisher's note: Springer Nature remains neutral with regard to jurisdictional claims in published maps and institutional affiliations. 


\section{References}

1. Siegel RL, Miller KD, Jemal A. Cancer statistics, 2019. CA Cancer J Clin. 2019;69:7-34.

2. Chen F, Roberts NJ, Klein AP. Inherited pancreatic cancer. Chin Clin Oncol. 2017;6:58.

3. Tamura K, Yu J, Hata T, Suenaga M, Shindo K, Abe T, et al. Mutations in the pancreatic secretory enzymes CPA1 and CPB1 are associated with pancreatic cancer. Proc Natl Acad Sci USA. 2018;115:4767-72.

4. Shindo K, Yu J, Suenaga M, Fesharakizadeh S, Cho C, Macgregor-Das A, et al. Deleterious germline mutations in patients with apparently sporadic pancreatic adenocarcinoma. J Clin Oncol. 2017;35:3382-90.

5. Raphael BJ, Hruban RH, Aguirre AJ, Moffitt RA, Yeh JJ, Stewart $\mathrm{C}$, et al. Integrated genomic characterization of pancreatic ductal adenocarcinoma. Cancer Cell. 2017;32:185-203.

6. Grant RC, Selander I, Connor AA, Selvarajah S, Borgida A, Briollais L, et al. Prevalence of germline mutations in cancer predisposition genes in patients with pancreatic cancer. Gastroenterology. 2015;148:556-64.

7. Waddell N, Pajic M, Patch A-M, Chang DK, Kassahn KS, Bailey $\mathrm{P}$, et al. Whole genomes redefine the mutational landscape of pancreatic cancer. Nature 2015;518:495-501.

8. Hu C, Hart SN, Polley EC, Gnanaolivu R, Shimelis H, Lee KY, et al. Association between inherited germline mutations in cancer predisposition genes and risk of pancreatic cancer. J Am Med Assoc. 2018;319:2401-9.

9. Schmitt A, Feldmann G, Zander T, Reinhardt HC. Targeting defects in the cellular DNA damage response for the treatment of pancreatic ductal adenocarcinoma. Oncol Res Treat 2018;41: 619-25.

10. Ayars M, Eshleman J, Goggins M. Susceptibility of ATM-deficient pancreatic cancer cells to radiation. Cell Cycle. 2017;16:991-8.

11. Teive HAG, Moro A, Moscovich M, Arruda WO, Munhoz RP, Raskin S, et al. Ataxia-telangiectasia - a historical review and a proposal for a new designation: ATM syndrome. J Neurol Sci. 2015;355:3-6.

12. Ahmed M, Rahman N. ATM and breast cancer susceptibility. Oncogene 2006;25:5906-11.

13. Roberts NJ, Jiao Y, Yu J, Kopelovich L, Petersen GM, Bondy ML, et al. ATM mutations in patients with hereditary pancreatic cancer. Cancer Discov. 2012;2:41-46.

14. Brand R, Borazanci E, Speare V, Dudley B, Karloski E, Peters MLB, et al. Prospective study of germline genetic testing in incident cases of pancreatic adenocarcinoma. Cancer 2018;124: 3520-7.

15. Roberts NJ, Norris AL, Petersen GM, Bondy ML, Brand R, Gallinger $\mathrm{S}$, et al. Whole genome sequencing defines the genetic heterogeneity of familial pancreatic cancer. Cancer Discov. 2016;6:166-75.

16. Goggins M, Offerhaus GJ, Hilgers W, Griffin CA, Shekher M, Tang D, et al. Pancreatic adenocarcinomas with DNA replication errors $(\mathrm{RER}+)$ are associated with wild-type K-ras and characteristic histopathology. Poor differentiation, a syncytial growth pattern, and pushing borders suggest RER + . Am J Pathol. 1998;152:1501-7.

17. Banville N, Geraghty R, Fox E, Leahy DT, Green A, Keegan D, et al. Medullary carcinoma of the pancreas in a man with hereditary nonpolyposis colorectal cancer due to a mutation of the MSH2 mismatch repair gene. Hum Pathol. 2006;37:1498-502.
18. Wilentz RE, Goggins M, Redston M, Marcus VA, Adsay NV, Sohn TA, et al. Genetic, immunohistochemical, and clinical features of medullary carcinoma of the pancreas: A newly described and characterized entity. Am J Pathol. 2000;156:1641-51.

19. NCCN Clinical Practice Guidelines in Oncology, Pancreatic Adenocarcinoma, [Internet], Version 1. 2019. National Comprehensive CancerNetwork, 2018, [cited 5 March 2019]. https://www.nccn.org/professionals/physician_gls/pdf/pancrea tic.pdf. 20

20. Richards S, Aziz N, Bale S, Bick D, Das S, Gastier-Foster J, et al. Standards and guidelines for the interpretation of sequence variants: a joint consensus recommendation of the American College of Medical Genetics and Genomics and the Association for Molecular Pathology. Genet Med. 2015;17:405-24.

21. Bosman FT, Carneiro F, Hruban RH, Theise N. WHO classification of tumours of the digestive system. 4th ed. France: IARC Press; 2010.

22. Basturk O, Hong S-M, Wood LD, Adsay NV, Albores-Saavedra $\mathrm{J}$, Biankin AV, et al. A revised classification system and recommendations from the Baltimore consensus meeting for neoplastic precursor lesions in the pancreas. Am J Surg Pathol. 2015;39:1730-41.

23. Matthaei H, Wu J, Dal Molin M, Shi C, Perner S, Kristiansen G, et al. GNAS sequencing identifies IPMN-specific mutations in a subgroup of diminutive pancreatic cysts referred to as "incipient IPMNs. Am J Surg Pathol. 2014;38:360-3.

24. Ryan R, Gibbons D, Hyland JMP, Treanor D, White A, Mulcahy $\mathrm{HE}$, et al. Pathological response following long-course neoadjuvant chemoradiotherapy for locally advanced rectal cancer. Histopathology 2005;47:141-6.

25. Protocol for the Examination of Specimens From Patients With Carcinoma of the Pancreas, [Internet], version PancreasExocrine 4.0.0.1. CAP, 2017, [cited 5 May 2019]. https://documents.cap. org/protocols/cp-gihepatobiliary-pancreas-exocrine-17protocol4001.pdf.

26. Kakar S, Pawlik TM, Allen PJ, Vauthey JN. Exocrine pancreas. In: Amin MB, Edge S, Greene F, Byrd DR, Brookland RK, Washington MK, et al., editors. AJCC cancer staging manual. 8th ed. New York, NY: Springer; 2017. p. 337-50.

27. Singhi AD, Ishida H, Ali SZ, Goggins M, Canto M, Wolfgang $\mathrm{CL}$, et al. A histomorphologic comparison of familial and sporadic pancreatic cancers. Pancreatology 2015;15:387-91.

28. Shi C, Klein AP, Goggins M, Maitra A, Canto M, Ali S, et al. Increased prevalence of precursor lesions in familial pancreatic cancer patients. Clin Cancer Res. 2009;15:7737-43.

29. Bannon SA, Montiel MF, Goldstein JB, Dong W, Mork ME, Borras E, et al. High prevalence of hereditary cancer syndromes and outcomes in adults with early-onset pancreatic cancer. Cancer Prev Res. 2018;11:679-86.

30. He J, Ahuja N, Makary MA, Cameron JL, Eckhauser FE, Choti MA, et al. 2564 resected periampullary adenocarcinomas at a single institution: trends over three decades. HPB. 2014;16:83-90.

31. Allen PJ, Kuk D, Castillo CF-D, Basturk O, Wolfgang CL, Cameron JL, et al. Multi-institutional validation study of the American Joint Commission on Cancer (8th Edition) Changes for $\mathrm{T}$ and $\mathrm{N}$ staging in patients with pancreatic adenocarcinoma. Ann Surg. 2017;265:185-91.

32. Desmet F-O, Hamroun D, Lalande M, Collod-Béroud G, Claustres M, Béroud C. Human Splicing Finder: an online bioinformatics tool to predict splicing signals. Nucleic Acids Res. 2009;37:e67. 